This is an electronic reprint of the original article. This reprint may differ from the original in pagination and typographic detail.

Author(s): Rantala, Katja; Karjaluoto, Heikki

Title: $\quad$ Value Co-Creation Opportunities : Managerial Transformation of Digitisation Risks into Success Factors

Year: $\quad 2018$

Version:

Please cite the original version:

Rantala, K., \& Karjaluoto, H. (2018). Value Co-Creation Opportunities : Managerial Transformation of Digitisation Risks into Success Factors. In P. N. Melo, \& C. Machado (Eds.), Management and Technological Challenges in the Digital Age (pp. 31-52). CRC Press. Manufacturing Design and Technology.

All material supplied via JYX is protected by copyright and other intellectual property rights, and duplication or sale of all or part of any of the repository collections is not permitted, except that material may be duplicated by you for your research use or educational purposes in electronic or print form. You must obtain permission for any other use. Electronic or print copies may not be offered, whether for sale or otherwise to anyone who is not an authorised user. 


\title{
Value Co-Creation Opportunities: Managerial Transformation of Digitisation Risks into Success Factors
}

\author{
Author(s): \\ *K. Rantala (Univ of Jyväskylä, rantakat@gmail.com) \\ H. Karjaluoto (Univ of Texas, heikki.karjaluoto@jyu.fi)
}

\section{x.1 Introduction: Rethinking Healthcare Services}

This chapter introduces the development work of digitized healthcare services through a case organization through value co-creation in networks. Further, as in healthcare the issue of quality is central in terms of risks, this study discusses through value co-creation elements the risks the management is facing in the digitization of services.

Transforming service into digital format has been ongoing in consumer service industries already for some time especially in areas of travelling, banking and even some governmental services like tax bureau. Logically healthcare sector is following this development as there seems to be a general expectation value for better availability, self-service and independency from the service provider (OECD, 2013; Martin et al., 2009). Digital solutions in healthcare are effectively utilized in medical and treatment processes through various equipment, but in terms of service and empowering and activating the customer, the development of digital services confronts totally new arena and even challenges of healthcare consumerism meaning the customer making active independent choices for the service or treatment (Fischer, 2014). Healthcare services are traditionally developed based on the development of science of medicine. This kind of development work is enhanced within the healthcare organization with some inputs by the patient through inquiries and the participation of any other organization in the development work is based mainly on the expertise in the area of medicine. For the development of services digitization opens up possibilities to increase the customer focus by involving the customer in the actual development work of the digital 
services and utilizing a network of actors in the development work each contributing with their respective expertise to the development work. These kinds of networks consist of organizations and actors that are oriented toward a mutual target with joint efforts. These organizational networks can be described as value networks creating social and economic good or value supported by exchange of information (Allee 2009, Vargo and Lusch, 2016)). With the public funding these networks of organizations consist of several interest parties all focused on collaborating with the development work of digital services. These networks are dynamic value co-creation configurations integrating resources of people and technology and are not only sharing, but accumulating information and knowledge to meet the joint target (Ostrom et al., 2015; Spohrer and Maglio, 2010; Vargo and Lusch, 2016).

Healthcare environment consists of complex interrelations and networks within an organization and between these organizational units. The complexity of the healthcare environment is challenging both for the operators within this environment, and for the citizens seeking their way to obtain care or professional help. The governmental actions to increase transparency of the healthcare services are part of the aim for open government (OECD, 2013; Greve, 2013). The open government further strives for accessibility and responsiveness of the public actions. These ideas of open government are applicable to public healthcare and carry with them similar dimensions as the idea of value co-creation.

\section{x.2 From Value Creation to Value Co-Creation}

Healthcare is a complex service system that implicitly strives for enhancing the public good and for this purpose integrates various resources of the service system. Healthcare is traditionally viewed as producing value. According to Vargo and Lusch (2916) value cannot be produced as the value is defined only by the beneficiary, and followingly the service provider can only offer value propositions. Value co-production carries the idea of exchange and a possession of a service and is hence derived from the service provider's context. Value 
co-creation is differing from co-production as value co-creation includes the elements of customer as an active actor and participant in the value co-creation process (Vargo and Lusch, 2008).

Moore (2000) presented the idea of public value creation. In public value creation the customers are the citizens that determine value. From the very nature value is something that cannot be defined or produced by the service provider (Siltaloppi \& Vargo, 2014). Along with service dominance logic value is created in-use differing from the idea of exchange based value, which refers to a utility against compensation (Grönroos and Voima, 2013). Value- in-use is the experiential value or perceived value based on the consumption of the service (or goods), which emphasizes the process nature of value creation. The perceived or experienced value is not static and can change during the process or differ from the expectation in the end of the process. In healthcare the customer perceived or experienced value follows the same logic and cannot be defined before usage of the service (Grönroos and Voima, 2013; Ranjan and Read, 2016; Vargo, Maglio and Akaka, 2008).

Value co-creation is defined to occur during interaction between the service provider and the customer. These two actors meet in a joint sphere for mutual interests (Grönroos and Voima, 2013). In healthcare the joint sphere of interaction is corresponding with the appointment of the doctor with the customer or the patient. This interaction traditionally is based on a faceto-face meeting, which is utilizing the scarce resources and has the element of informing the customer or patient of the condition with relevant, but limited information. As healthcare professions are highly specialized, regulated and legitimized for diagnosis, the customer is not in the position to acquire or possess information related to the medical or other health condition in a way that would make both parties equal and the interaction balanced. Logically then, the service provider or the doctor is in a strong position to contribute to the value cocreation process greatly with the knowledge, information and resources at hand and support 
the value creation of the customer. The imbalance of the relationship in terms of knowledge may cause difficulties for value co-creation as customers are lacking the information and their value perceptions may be influenced by the way the service provider presents the service offering (Edvardsson \& al., 2011). In practice this would require the professional to meet the customer more than half the way in interaction and hence support the customer in the value co-creation process.

\section{x.3 Digitization Creating Value Co-Creation Opportunities}

Value co-creation between the service provider and the customer takes place in the joint sphere of interaction through dialogue with the use of digital solutions acting as platforms or mechanism for this dialogue and value co-creation (Grönroos and Voima, 2013; Saarijärvi, Kannan and Kuusela, 2013). Both the service provider and the customer can utilize their networks or independent spheres for value co-creation and this value co-creation can also be independent of the service provider's intervention or facilities like in case of customer's sphere (Grönroos and Voima, 2013; Ranjan and Read, 2013; Ordanini and Pasini, 2008; Grönroos and Ravald 2011).

Digitalization of the service creates possibilities for temporal and spatial service separation and hence, a completely new arena for the value co-creation process with engaged and empowered customer with independent networks in customer's sphere. The service separation is enabled with the digital service platform that can be accessed from anywhere at anytime, differing completely from the traditional appointment with the doctor at the doctor's facilities. The temporal separation means that the dialogue can have a timewise continuum where both the customer and the service provider utilize the possibilities of the services portal regardless whether the other party is on-line or not. In other words, instead of the traditional way of dialogue during the direct appointments, dialogue becomes a process supported by the digital portal with messaging, chat or independent service actions within the portal. Further 
the spatial separation of the service interaction is enabled through the digital platform. The digital service can be accessed at anytime from anywhere and is not bound to any specific location or facilities of the service provider. Through this separation of service digitalization transforms the interaction of the acting parties and emphasizes the process nature of the service supported by the service portal. Through the service separation the digitization of the service increases the availability and access to the service, which ultimately means that the unmet needs can be better met as the service is scalable in a magnitude not possible with the traditional appointments.

The concept of value co-creation is complex and still is calls for a consistent theory or evolution (Ranjan and Read, 2014; Grönroos and Helle, 2012; Vargo and Lusch, 2016). Therefore the operationalization of the concept is more meaningful for the purpose of this chapter. This chapter focuses on discussing the operationalization of the value co-creation through digitization with DARIO model further developed on the work of Prahalad and Ramaswamy (2004) of building blocks of value co-creation, the so called DART model. The letters of DART stand for dialogue, access, risk-benefits and transparency.

DARIO model (see Figure 1) combines the four building blocks with digitization and opens further the concept of transparency into transparency of information and operations in form of standard work introduced by defined digital work processes. With these elements of dialogue, access, risks, information transparency and operations DARIO model introduces the idea of value co-creation opportunities through digitization of services.

Figure 1: DARIO model of value co-creation in digitized services Offering value co-creation opportunities or enabling them is applicable in healthcare as the service provider has the access to knowledge and has the legitimized position meaning that the responsibility of the service provider is emphasized in relation to the customer as these two cannot meet on equal terms (Edvardsson and al., 2011). This means that the service 
provider enables and greatly contributes to the value co-creation and hence offers opportunities for value co-creation. The shared information and jointly set targets for the care or treatment and the active participation of the customer in decision making and treatment are contributing to the process and end result. The customer becomes an active participant in shared decision making with shared information and targets for the treatment or service (Gionfriddo \& al., 2013; Hoffmann, 2014; Carman and Workman, 2017), which consist of dialogue and mutual interests, crucial elements of value co-creation.

Digitization from its very nature is a disruptive factor for a service process and this disruption carries the element of uncertainty or risk within. Prahalad and Ramaswamy (2004) have considered the risks of the value co-creation process from the customer point of view. The customer may be puzzled with for example questions of medication and its risks and is looking for answers through dialogue (Prahalad and Ramaswamy, 2004). In the DARIO model the risks-benefit is discussed from the service provider's perspective with the concept of risks related to the integration of the digital service with the overall service process. The risks in healthcare are of great concern and very much connected to the quality of the service. The medical service quality is undoubtedly of major concern and here the involvement of the professionals contributing to the development work of digital service content is of great importance. However, the risks related to digitalization are much more complex as they not only concern the medical service quality, but also the risks of the development work, integration, utilization and acceptance of the digital services. The internal risks related to the utilization or integration of the digital service within the service process and acceptance to use the digital service portal in the customer interface present risks where the internal actions of the organization contribute greatly to the outcome (Sykes, Venkatesh and Gosain, 2009). There is less extensive research on the digitization of health care services from the implementation perspective, but there are studies on implementing electronic medical 
records. These studies have been analyzed in a study by $\emptyset$ vretveit \& al. (2007) and the study identifies differences in how the work processes as redesigned, either as part of implementation or as a benefit. According to this study repeated factors can be found for successful implementation. Other than technical system related features, these factors encompass features of the implementation process, leadership, resources and the organization's culture. The impact of the organizational culture and collaboration is realized either in improved or reduced cooperation. The successful integration of the digital service with the overall service process creates value co-creation opportunities. The integration is enhanced through defined working processes of operations in the implementation. If the integration of digital services with the overall service process fails in the implementation, there is less possibility to reach proper acceptance for a digital service by the professionals and effective utilization may fail due to complex working processes. Logically, the technology acceptance process and the impact of peer support are of significant relevance when integrating the service and exploiting the possibilities engaged (Sykes, Venkatesh and Gosain, 2009).

\section{x.4 Developing Digital Services for Healthcare}

This chapter describes development and its challenges of digital healthcare services of a public healthcare organization. The organization is developing new digital services as well as transforming services on digital platform. The development work has started years ago within the organization. The development work is on-going on several medical fronts, but the starting point has been the development of digital services and service platforms of mental therapy and weight control services. The development work has been carried out by the organization's professionals of these respective areas with the help of ICT department. The path has been long and the current success and acceptance of these digital services has required great enthusiasm and commitment. Further, currently as other similar services are 
being developed, the work is involving a network of other organizations and actors contributing to the development work.

This chapter is based on findings of the two pioneering applications of mental therapy and weight control services in digital format. The data is gathered by multiple interviews, focus group interviews and through observations within the organization as one of the authors working within the organization. The observations are based on seminars and workshops of the organization dealing with digitization and digital services. The interviews, both individual and focus group interviews, were thematic interviews based on DART model of Prahalad and Ramaswamy (2004). The individual interviews were carried out with snowball sampling (Salganik \& al., 2004) where each interviewee identified the next participant. The focus group interviews were mix of people of the organization keenly involved with the development work and digitization of healthcare services, not specific on the mental therapy or weight control services. For this specific chapter the interviews were further replenished to focus more closely on the risks of digitization based on the DARIO model of value cocreation opportunities. The purpose was to enlarge the comprehension of the prevailing risks for the service provider and for the management responsible for implementing these services. Further the observations were replenished with an additional workshop on risks related to technology and digitization organized by the ICT department of the organization. Both these case services of mental therapy and weight control are well advanced and of special healthcare quality. Both consist of three separate sections first being the general section of information on the topic of mental conditions and issues of weight control and obesity for anyone to access. This service portal also offers the possibility for self-navigating and assessing the options for service. The second section of these service portals is aimed for the actual therapy, which requires a referral to be entered. The actual therapy part is then opened when the referral is sent and accepted. The therapy planning is done jointly with the 
customer and the information during the therapy is stored within the portal and is accessible at anytime by participants. Participants for the therapy are the therapist and the customer. The therapy is working on consulting basis and the responsible physician will receive the report after the therapy is finalized. Further, the customer can choose how much of the therapy process the customer will share with the responsible physician. The therapy is steered by algorithms and the process is normally planned for one year having a starting, mid-process, and finalizing reviews with the therapist. If the customer wishes additional virtual appointments can be agreed upon. The third section of the service platform of these therapies is for the professionals, who can enter for information and further contribute to the development of the service and content. The information offered in all these sections is of special healthcare professional quality, evidence-based and is constantly updated with the latest research available.

The digital service portal supports the interaction through-out the service process duration with available, stored and shared information. The transparency of the information is brought to a completely new level through the digital service portal. The customer related information is stored in the service portal during the service process and is accessible at anytime by both parties, the customer and the service provider. Further, the service provider with the help of the network of professionals contributing to the service development and maintenance is providing evidence based high level professional information for the customer. The information is freely accessible within the service for supporting self-care tasks, providing correct, evidence based information for the customer condition or need for support through correct information.

The customer empowerment is enabled by the service provider's actions in the service provider's sphere. The digital service format enforces the service provider to follow a predefined, more standardized way of working with the service portal. This standardized way 
of working appears for the customer in the joint-sphere as transparency of the service process. The customer is involved from the beginning in the design of the service process that has jointly agreed targets and a predefined duration. This is dramatically different form the traditional appointment based service, where there is missing and loss of information between the appointments and the customer learns only step by step the next phase of the service. The digital therapy service is based on agreed targets and transparent process that is supported by the digital portal with self-care tasks for the customer in various phases of the service.

The possibility to access the service is multiplied in the digital service format when compared to the traditional appointments. A therapist for example in case of the mental therapies can take five to six patients or customers through a scheduled appointment. With the digital service one therapist can have over twenty customers a day. This increase in availability of appointment times due to the efficient service structure increases the access to the service tremendously. This can have dramatically different influences for the population as the unmet needs for therapy can increasingly be met. The service structure engages the customer with tasks that traditionally would consume the therapist time in the appointment session. Now the customer can contribute independently to the care process with tasks of providing information and self-care tasks.

Healthcare industry is strictly regulated with licensed professions and legislation for the patient security and safety. This emphasis the role of the service provider in assessing and taking the risks of digitalization into account.

\section{x.5. Collaboration in Value Co-Creation on Digital Services}

The development of value co-creation theory is moving from dyadic perspective towards a network orientation. The idea of integrating micro-specializations is changed to a more generic description of multiple, economic and social, actors as resource integrations (Vargo and Lusch, 2016). Technical or digital solutions can act as mechanisms through which 
additional resources are integrated for value co-creation processes (Saarijärvi, Kannan and Kuusela, 2013). These additional resources consist of network of organizations and other actors that contribute to the development work of these digital services and enable the construction of value co-creation opportunities. The network of professionals makes a major contribution to the development work providing and sharing the special healthcare level of information that is widely opened through these services to primary care physicians, therapists and customers. Hence, in this network of actors the service provider is integrating various resources like knowledge to support the customer in value creation process (Edvardsson et al, 2011).

In the development teams of the case organization there are multiple organizational actors participating and giving their input and expertise of the actual medical science in use. There seems to be a lot of willingness to contribute to the digitization of the services, but there is an evident need for clear strategy as there are comments from the participating parties that their input is of minor value and hence, the network is not comprehensively included in the development work, or there is no clear target for implementation of the services, and even worse there seems to be unawareness of the actual implemented services available. There are plenty of start-up entrepreneurs that are willing to offer different kind of solutions and gadgets for healthcare services during this ongoing booming transformation of services. For the management the challenge is to find right partners that have commitment to the development work required. The development work of digital healthcare services encompasses enormous efforts to ensure quality, security and safety aspects. This proceeds high level of commitment in the development work, which requires continuous resourcing in terms of capabilities, financing and time. Further, as the connectivity issue among various ICT systems is critical for the service process, the various applications and software offered causes multiple problems with the connectivity issue and hence due to resources the 
possibilities to engage perhaps attractive software may be limited. Therefore, the case organization has chosen the way to take a lead itself and start developing digitized healthcare services that are of high quality from the content point of view as well as from the connectivity point of view.

There is a call for marketing these digitized services among participating actors in the networks. The participation into the development work reaches only a small fraction of the organizations and the general knowledge of opportunities is not reaching the final users from the professionals as well as from the customer side. Perhaps a more effective solution would be the closer involvement of the network participants in the strategy development of digitization and target setting for the implementation of these digital services. The network actors are not closely connected or discussing actively shared view of the strategy although the exists government level of commitment to the development work in terms of financing. This would require stronger management level involvement of the participating network organizations in defining the strategic targets with service architecture, work processes and procedures in order to draw full benefits of a wide digital service offering for various medical expert areas under development.

Digitalization increases availability and enables sharing of information, as components of value co-creation, in such a magnitude that it is said to revolutionize healthcare as it fulfils the unmet needs of the public for healthcare. But because health-care services are strictly regulated, it is essential that the digital services should be carefully analyzed before entering the digitization process so that their role in the service architecture can be evaluated. The case organization is proceeding simultaneously with service architecture design, service design and developing digitalized services at a very wide front with various actors and networks. There seems to be intra-organizational challenges between actors involved with the service architecture design and the actual digital service development work. The part of organization 
that is working to design a holistic service architecture for the organization is separate from the part of organization that is actively developing the digital services and not connected to the network of actors consisting of multiple organizations in the development work. The challenge seems to be on the interaction of the architecture design and designing new emerging work processes generated by the digital service platforms.

\section{x.6. Management Coping with the Risks of Digitization}

The development of the digital services comes from two approaches; transferring present services into digital format and describing the new processes accordingly and developing services that are actually quite new and possible through digitization. The service design is based on the work in these development working groups, but the connection to the practical processes of the professionals is not tightly linked into the development work due to voluntary participation and missing jointly agreed and set targets for implementation. The overall service architecture, service design and the development of the actual digital services proceed parallel, but the coordination is very much on responsibility of ICT dedicated for this service development work and the management is not systematically adhered to it.

ICT security procedures and legislation are the directing guidelines in the development work for the organization. The legislation and EU directives define the digital healthcare service platform as a medical device, which sets the foundation for the testing and acceptance procedure of the service. The organization utilizes risk analysis in evaluating the service platform from the ICT security perspective and from the managerial usability perspective. The content of the risk analysis is defined based on the purpose of the analysis. For example the usability has different risk evaluation than when analyzing the software application data security.

The ICT development work is based on life-cycle thinking of the digital service software and ICT platform and ICT services. The planning, actual development work and implementation 
phases are considered from the risk perspective to secure continuity and availability of the service and a proper handling of disturbances. This is covered by having generic elements in the digital services and then separate features of the service content.

The technical risk related to the service application or networks is connected with the ICT, but there seems to be a division of responsibility concerning these new digital services of the organization. The ICT department takes the responsibility of the basic infrastructure, but the functioning of the digital services and their applications and networks are pointed to the development team of ICT, which from a holistic perspective of risk management is perhaps not a sustainable risk management practice. Not only the practical implementation related risks emerge, but the risks for the information security and risks for the usability of the service arise. It is on the responsibility of the service provider to secure the service platform in a way that the information and usability are not endangered. The service provider in the customer interface is the physician or the therapist and in this interface there needs to be clear responsibility and security action plan for cases when the service is not available and the customer is suffering for lacking the therapy or contact possibility with the therapist through the digital service platform. This traditional responsibility of the service by the physician in the customer surface causes the attitude within professionals that the fear for errors or mistakes becomes a hinder for the digitization of services and innovations. The fear for shift of responsibility creates unnecessary blockages as the digitization does not outsource the responsibility of the physician nor does it delude the process, on the contrary the digitized service process is transparent also for the physician. "The professionals need to change and review processes from another perspective" (Project director, ICT).

The identified features for successful implementation (Øvretveit \& al., 2007) are identifiable risk areas in the implementation of these digital healthcare services. For a successful value co-creation these risks involved with the implementation process, leadership, resources and 
organizational culture are covered in the risk analysis idea, but there seems to be a gap in operationalizing the measures to prevent the realization of the risks.

Based on the risk analysis findings of resources already in the developing phase of the services, there is an imminent risk of inadequate capabilities of required respources concerning digital solutions. Further the attitude of the management of the organization allocating these resources may not be supportive and that the digitization is regarded by the organization something like "a compulsory trouble". These risks of lacking capabilities and adverse attitudes slow the starting process of the development work when resources are being acquired. Hence, the project organization strives for to create an understanding of the magnitude of the transformation required and that with the current traditional ways of working, equipment, resources and knowledge of digitization, the progress in the development work requires time and allocated resources from the organization accordingly. "This is a journey to understanding and realizing of what could be" (Project director, ICT). Further managerial risk is there that the personnel cannot keep up with the speed of change and resistance hinders the successful implementation. This requires the adaptation of the development speed with the engagement of the personnel. The target is to involve the personnel to develop their daily work processes with the digital service by defining the benefits the digitalization can bring to the work and for the customer. The capabilities of the organization to support the implementation are mapped and supported with targeted measures one being the standardization of the service. Despite the target of standardization, there is no fixed development formula for this and the development work is done tailoring each service separately. This has evident challenges and is not always successful as the management culture is not necessarily adapting with the use of the digital services. It is crucial how the management understands the change and the imposed requirements of digitization for the work processes and managing of the work and personnel. "The digitized service brings the 
customer more involved with the service process and the role of the customer is increasing. For the service organization unit this means a tremendous change in the service culture, which should be apparent and visible in each customer interaction and in the entire service process". (Project Director, ICT)

The transformation of the services into digital format requires a wide understanding and knowledge of the work processes related. This introduces the need for new definitions of work processes and a service architecture that combines the various ways of offering the service to the customer. Finally in the customer interface it is the physician who has to be able to support the customer in the choice of a proper service method and its content. This capability of the physician requires training for the new digital services and for the new service architecture and the linkages between the service elements of the digital and traditional format of the service. Digitization of the services resulting in changing work processes and habits evidently will change the working culture and the company culture. Logically, this brings in the challenge that the management is facing with the practical implementation of these digital services with applicable, defined and integrated, but missing work processes.

Further, digitization causes a dilemma with networks of multiprofessional teams and collaboration. The digital processes with the customer are based on dual interaction and the shared information cannot be distributed to any third party unless the customer so wishes. Still for the overall service process there is the need for multiprofessional teams engagement and expertise like in case of weight control processes. The customer may start the process to treat obesity with tasks and acquiring information targeted to prepare the customer for an obesity surgery. After the surgery the customer and the health condition requires other professionals to support the recovery process, nutrition change necessities and perhaps even related mental issues. Combining this need multiprofessional work with a digital service 
requires a change in working culture and also in the work processes. This is not still closely planned and defined with the development work and requires a lot of managerial input with the development teams for a successful end result. The definition responsibility of the new working processes easily remains on individual managers of operations that struggle with the professionals' acceptance of new ways of working and the combining the need for multiprofessional teams work with new processes.

\section{References}

Allee, Verna. 2009. "Value-creating networks: organizational issues and challenges". The learning organization 16, no.6: 427-442.

Carman, Kristin L., and Thomas A. Workman. 2017. "Engaging patients and consumers in research evidence: applying the conceptual model of patient and family engagement”. Patient education and counseling 100, no.1:25-29.

Edvardsson, Bo, Bård Tronvoll, and Thorsten Gruber. 2011. "Expanding understanding of service exchange and value co-creation: a social construction approach". Journal of the Academy of Marketing Science 39, no.2:327-339.

Fischer, Sophia. 2014. "Hospital Positioning and Integrated Hospital Marketing Communications: State-of-the-Art Review, Conceptual Framework, and Research Agenda”. Journal of Nonprofit \& Public Sector Marketing 26, no.1:1-34

Gionfriddo, Michael. R., Aaron,L. Leppin, Juan, P. Brito, Annie LeBlanc, Nilay, D. Shah, and Victor, M. Montori. 2013. "Shared decision-making and comparative effectiveness research for patients with chronic conditions: an urgent synergy for better health". Journal of Comparative Effectiveness Research 2, no.6:595-603.

Greve, Carsten. 2015. "Ideas in public management reform for the 2010s. Digitalization, 
value creation and involvement”. Public Organization Review 15, no.1: 49-65.

Grönroos, Christian, and Pekka Helle. 2012. "Return on relationships: conceptual understanding and measurement of mutual gains from relational business engagements". Journal of Business \& Industrial Marketing 27, no.5:344 - 359.

Grönroos, Christian, and Annika Ravald. 2011. "Service as business logic: implications for value creation and marketing". Journal of Service Management 22, no.1:5-22.

Grönroos, Christian, and Päivi Voima. 2013. "Critical service logic: making sense of value creation and co-creation". Journal of the Academy of Marketing Science 41, no.2:133150.

Hoffmann, Tammy C., Victor M. Montori, and Chris Del Mar. 2014. "The connection between evidence-based medicine and shared decision making". Jama 312, no.13:1295-1296.

Martin, Graham, Graeme Currie, and Rachael Finn. 2009. "Reconfiguring or reproducing intra-professional boundaries? specialist expertise, generalist knowledge and the 'modernization' of the medical workforce." Social Science \& Medicine 68, no.7: 11918.

Moore, Mark H. 2000. "Managing for value: Organizational strategy in for-profit, nonprofit, and governmental organizations". Nonprofit and Voluntary Sector Quarterly 29, no.1:183-208.

OECD E-Government Project. 2013. "Draft OECD Principles for Digital Government Strategies.” Paper presented at the OECD E-Leaders' meeting, Bern, Switzerland, October 29-30.

Ordanini, Andrea, and Paolo Pasini. 2008. "Service co-production and value co-creation: The 
case for a service-oriented architecture (SOA)". European Management Journal 26, no.5: 289-297.

Ostrom, Amy. L., A. Parasuraman, D.E. Bowen, L. Patricio, and C.A. Voss. 2015. "Service research priorities in a rapidly changing context". Journal of Service Research 18, no.2:127-159.

Prahalad, Coimbatore, and Venkat Ramaswamy. 2004. "Co-creation experiences: The next practice in value creation." Journal of Interactive Marketing 18, no.3: 5-14.

Ranjan, Kumar Rakes, and Stuart Read. 2016. "Value co-creation: concept and measurement”, Journal of the Academic Marketing Science 44:290-315

Saarijärvi, Hannu, P.K.Kannan, and Hannu Kuusela. 2013. "Value co-creation: theoretical approaches and practical implications”. European Business Review 25, no.1: 6-19.

Salganik, M. J., and D.D.Heckathorn. 2004. "Sampling and estimation in hidden populations using respondent-driven sampling”. Sociological Methodology 34, no.1:193-240.

Siltaloppi, Jaakko, and Stephen L. Vargo. 2014. "Reconciling Resource Integration and Value Propositions--The Dynamics of Value Co-creation”. System Sciences (HICSS), 2014 47th Hawaii International Conference on. IEEE, 2014.

Spohrer, James C., and Paul P. Maglio. 2010. "Toward a science of service systems," in Handbook of service science, edited by Paul Maglio, 157-194. Springer US.

Sykes, Tracy Ann, Viswanath Venkatesh, and Sanjay Gosain. 2009. "Model of acceptance with peer support: A social network perspective to understand employees' system use." MIS Quarterly 33, no.2:371-93.

Vargo, Stephen, and Robert Lusch. 2008. "Service-dominant logic: Continuing the evolution." Journal of the Academy of Marketing Science 36, no.1: 1-10. 
Vargo, Stephen, and Robert Lusch. 2016. "Institutions and axioms: an extension and update of service dominance logic”. Journal of the Academy of Marketing Science 44: 5-23.

Øvretveit, John, Tim Scott, Thomas G. Thomas G. Rundall, Stephen M. Shortell, and Mats Brommels. 2007. "Improving quality through effective implementation of information technology in healthcare". International Journal for Quality in Health Care, 19, no5:259-266.

\section{Figure Legend}

Figure 1: DARIO model of value co-creation in digitized services. (Based on Prahalad and Ramaswamy 2004) 
Tähän kuvaan on viittaus tekstissä ja tämä tulee pdfnä tekstin mukaan erillisenä filenä.

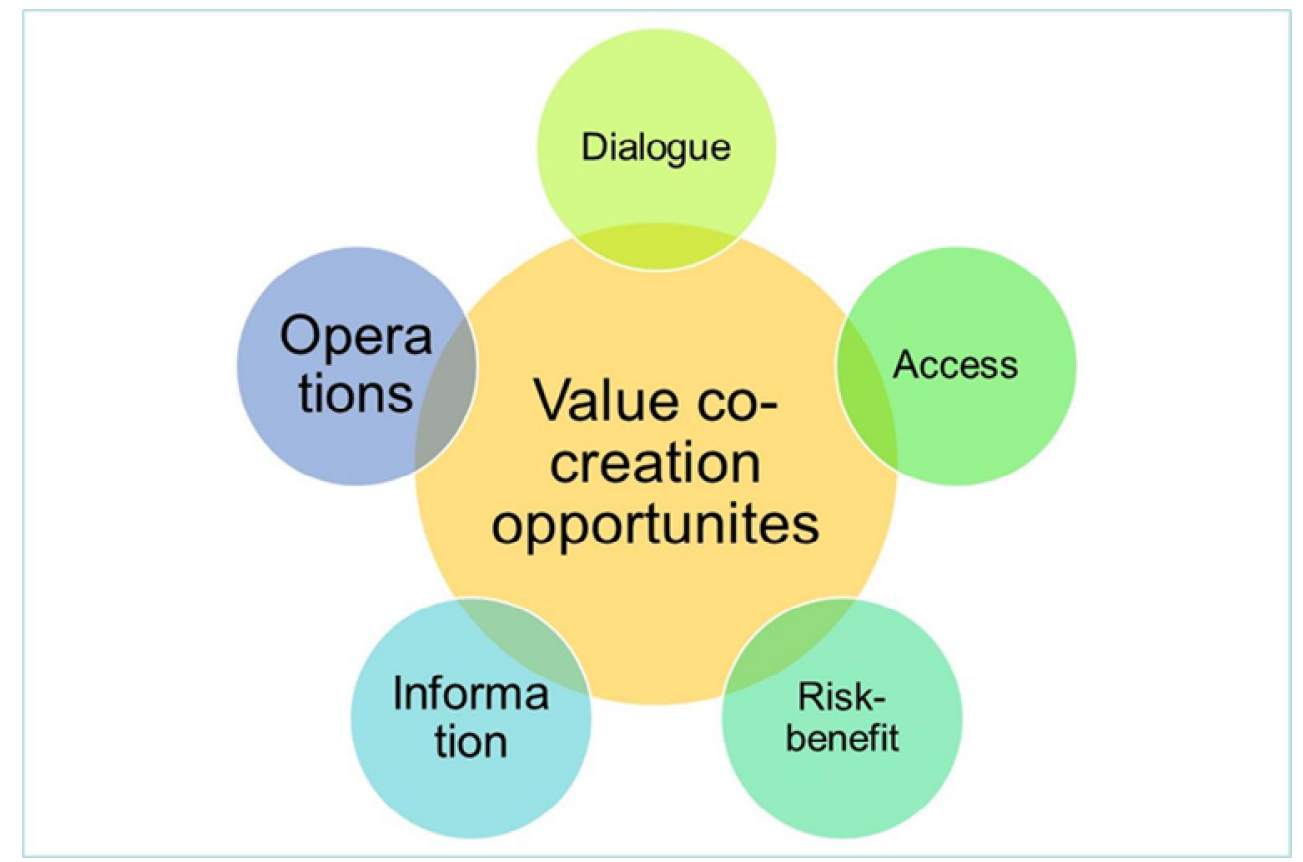

